\title{
KDR Mutation: A High-Frequency Rare Mutation and its Correlation with other Somatic Mutations in Indian Colorectal Cancer Patients
}

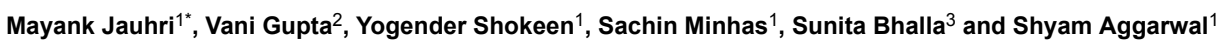 \\ ${ }^{1}$ Department of Medical Oncology, Sir Ganga Ram Hospital, New Delhi - 110060, India \\ ${ }^{2}$ Department of Biotechnology, Delhi Technological University, New Delhi-110042, India \\ ${ }^{3}$ Department of Pathology, Sir Ganga Ram Hospital, New Delhi - 110060, India
}

"Corresponding author: Mayank Jauhri, Department of Medical Oncology, Sir Ganga Ram Hospital, New Delhi - 110060, India, Tel: +91-9999991433; E-mail: mayank.jauhri@yahoo.com

Rec date: July 06, 2017; Acc date: July 25, 2017; Pub date: July 27, 2017

Copyright: (c) 2017 Jauhri M, et al. This is an open-access article distributed under the terms of the creative commons attribution license, which permits unrestricted use, distribution, and reproduction in any medium, provided the original author and source are credited.

\begin{abstract}
Aim: This study aims to find out the frequency of KDR mutation in colorectal cancer patients and if any correlation exists between KDR mutation and demographical features or with other common and uncommon gene mutations occurring in colon cancer.

Methods: FFPE samples of 112 patients were analyzed using next generation sequencing.

Results: KDR gene was found to be mutated most frequently $(19.6 \%)$ as compared to other uncommon gene mutations occurring among patients. 21/22 patients had the p.Q472H type of KDR mutation. It was significantly associated with mutations in PTEN $(p=0.003)$, KRAS $(p=0.026)$, APC $(p=0.033)$, EGFR $(p=0.036)$, NOTCH1 $(p=0.029)$ and ERBB4 $(p=0.008)$ mutations. More number of males $(22.06 \%)$ harbored KDR mutations than the number of females $(15.9 \%)$. A higher number of KDR mutations in Stage III $(31 \%)$ as compared to other stages - I-II $(19.23 \%)$ and IV $(7.31 \%)$ was reported. KDR mutations were found to be in greater number in patients with lymph node metastasis $(27.3 \%)$ as compared to liver metastasis $(8 \%)$. However, a statistically significant association of any clinical parameter was not found.
\end{abstract}

Conclusion: Our results suggest that although KDR is a rare somatic mutation in CRC, it plays a pivotal role in the development of colon cancer via angiogenesis pathway.

Keywords: Colorectal cancer; KDR; Mutation; Next generation sequencing; Rare mutation

\section{Introduction}

According to World Cancer Reports 2014, cancer accounted for 8.2 million deaths in year 2012 where colorectal cancer (CRC) was the third most common cancer in men and the second most common in women worldwide [1]. Also, as per the statistics given by Indian Council of Medical Research, CRC incidence in India is reported to be 4 patients per every hundred thousand people. CRC is a fatal disease caused and affected by various genetic mutations in biological pathways. This has been shown in KEGG (Kyoto Encyclopedia of Genes and Genomes) pathway database, that colorectal cancer is associated with and occurs via various biological pathways like Wnt, MAPK, PI3K, mTOR, VEGF, cAMP, p53 and other signaling pathways. These pathways may affect the processes of angiogenesis, gene repair, apoptosis, proliferation and differentiation.

Although all other pathways are equally crucial in development of CRC, VEGF pathway is mainly associated with angiogenesis and is responsible for its regulation in tumors. VEGF Pathway is followed through different signaling cascades depending on the type of signaling stimulus received by VEGF receptors, and thus leading to proliferation, migration, permeability or survival [2]. Any mutation in the proto-oncogenes involved in these pathways may lead to cancer by transformation into oncogenes. The VEGF signaling pathway has a key element namely KDR, also known as VEGFR-2 (Vascular Endothelial Growth Factor Receptor-2), stands for Kinase Insert Domain Receptor for VEGF-A. It is known to be a proto-oncogene, thus, affecting tumor angiogenesis. KDR gene is located on chromosome 4 at location $4 \mathrm{q} 12$. As per Cancerindex.org and Cancer Genetics Web, KDR gene mutation has been previously reported to be associated with lung and breast cancers. Importantly, KDR gene has been previously shown to play an important role in Cancer development via induction of vascularity, metastasis, apoptosis and proliferation in human colon cancer and breast cancer $[3,4]$.

Thus, it highlights the fact that CRC is well known to be associated with and caused by mutations and epigenetic alterations in certain common as well as rare genes regulating cellular signaling pathways like KRAS, BRAF, PIK3CA, KDR, PTEN, VHL, SMAD4 and others leading to cell proliferation and metastasis. The association of the disease with commonly occurring genetic mutations like those in $K R A S, B R A F$ and PIK3CA has been shown in various studies [5-7] but less attention has been given to the role and correlation of genetic mutations which occur rarely such as those in KDR, PTEN, SMAD4 and others. For such a heterogeneous disease, insights into the role of a rare mutation, in this case - KDR, can open new opportunities for treatment based research with KDR as a novel target. Therefore, in this study, we aim to find out frequency of KDR mutation in patients and if 
Citation: Jauhri M, Gupta V, Shokeen Y, Minhas S, Bhalla S, et al. (2017) KDR Mutation: A High-Frequency Rare Mutation and its Correlation with other Somatic Mutations in Indian Colorectal Cancer Patients. Next Generat Sequenc \& Applic 4: 148. doi: $10.4172 / 2469-9853.1000148$

Page 2 of 7

any correlation exists between KDR and demographical features or between KDR and other genes.

\section{Material and Methods}

\section{Patients and samples}

This study was initially conducted with 146 patients admitted at Sir Ganga Ram Hospital, New Delhi but after tumor content estimation only samples from 112 CRC patients remained. Clinical features of the patients including gender, age, anatomical site, depth of invasion, tumor size, distant metastasis etc. were recorded. December 2015 was the Follow-up end point of the patients under study.

Formalin fixed paraffin embedded (FFPE) blocks, obtained from Department of Histopathology, with tumor content $>30 \%$ were used for the study and those rejected were because they were old tissue blocks. Microtome was used for extraction of DNA from five to eight sections of $10 \mu \mathrm{m}$ each. The DNA was isolated using Qiagen ALL Prep DNA FFPE kit following manufacturer's instructions.

\section{NGS analysis}

All isolated dsDNA (double stranded DNA) samples and protein contamination were quantified using Nanodrop and QuBit respectively. The DNA samples with 260/280 ratio between 1.7-2.0 were selected for the study. For further qualification, IlluminaInfinium Assay was performed and only those samples having delCT value less than or equal to 2 were chosen. Sequencing library was created using 200-300 ng of dsDNA added to True Seq Amplicon Cancer Panel (Cat. No. FC-130-1008, Illumina) containing a 48 gene panel. Cancer Panel involved 212 amplicons of length 170-190bp which targeted hot spot mutations in 48 cancer related genes. DNA library was denatured, diluted and processed according to Illumina Protocol. MiSeq Reagent Kit V2 (Cat. No. MS-102-2002, Illumina) and MiSeq Reporter (V2.6.2, Illumina) were used to run samples, generate trimmed FASTQ files and align reads against whole genome build hg19.

Strand NGS V2.1.6 was used for further analysis and filtering of reads. The sequencing depth was 200x and target coverage was 5000x. Filtering out of read was done on the basis of failed vendor QC, average quality less than 20, alignment score below 90 and those with ambiguous characters. For detection of variants at locations in target regions covered by a minimum of 10 reads with at least 2 variant reads and variants with a decibel score of at least 50, Strand NGS variant caller was used. Substitution variants with StrandBias greater than $100 \%$ and InDel variants in homopolymer stretches greater than 4 with supporting reads of less than $10 \%$ were filtered out. 'SNP Effect Analysis" functionality in Strand NGS was used for analyzing the effect on protein and final variants were annotated using COSMIC (Catalogue of Somatic Mutations in Cancer) database.

\section{Statistical analysis}

SPSS 17.0 software was used for the analysis of data. Analysis was performed on two main categories of data; comparison of different clinical characteristics with mutations and co-existence of two or more genes together. Pearson's Chi Square test, Chi-square test or Fischer test of association were used to determine the relation between the above mentioned groups. $P$ value of less than 0.05 was considered to be statistically significant.

\section{Results}

In the current study, 112 CRC patient samples were analyzed for mutations occurring commonly and uncommonly in 48 genes. The total number of patients characterized according to their demographical characters is given in Table 1 . We calculated the per capita statistics of each clinical parameter for all patients having KDR mutation. It is noteworthy that more number of males $(22.06 \%)$ harbored KDR mutations than the number of females (15.9\%). KDR mutation might be associated with people with age $>60$ as these people were found to have higher number of KDR mutations $(23.07 \%)$ as compared to those with age $\leq 60(19.2 \%)$. KDR mutation was found to occur more frequently in Rectum (25\%) than in Colon (18.5\%).

\begin{tabular}{|c|c|c|c|}
\hline Clinical Parameters & $\begin{array}{l}\text { Total no. of } \\
\text { patients } \\
(n=112)\end{array}$ & $\begin{array}{l}\text { No. of patients } \\
\text { with } \\
\text { mutation }\end{array}$ & $(\%)$ \\
\hline \multicolumn{4}{|c|}{ Sex } \\
\hline$M$ & 68 & 15 & $22.06 \%$ \\
\hline $\mathrm{F}$ & 44 & 7 & $15.91 \%$ \\
\hline \multicolumn{4}{|c|}{ Age } \\
\hline$\leq 60$ & 73 & 14 & $19.18 \%$ \\
\hline$>60$ & 39 & 9 & $23.07 \%$ \\
\hline \multicolumn{4}{|c|}{ Anatomical site } \\
\hline Colon & 92 & 17 & $18.48 \%$ \\
\hline Rectum & 20 & 5 & $25.00 \%$ \\
\hline \multicolumn{4}{|c|}{ Location } \\
\hline Right & 46 & 13 & $28.30 \%$ \\
\hline Left & 46 & 8 & $17.40 \%$ \\
\hline \multicolumn{4}{|c|}{ Stage } \\
\hline$|-| \mid$ & 26 & 5 & $19.23 \%$ \\
\hline III & 45 & 14 & $31.11 \%$ \\
\hline IV & 41 & 3 & $7.31 \%$ \\
\hline \multicolumn{4}{|c|}{ Depth of invasion } \\
\hline $\mathrm{T} 2$ & 8 & 3 & $37.50 \%$ \\
\hline T3 & 75 & 14 & $18.67 \%$ \\
\hline $\mathrm{T} 4$ & 79 & 5 & $6.33 \%$ \\
\hline Metastasis & -- & 22 & -- \\
\hline Liver & 25 & 2 & $8.00 \%$ \\
\hline Lymph node & 22 & 6 & $27.30 \%$ \\
\hline \multicolumn{4}{|c|}{ No. of Nodes } \\
\hline No $(0)$ & 34 & 5 & $14.70 \%$ \\
\hline N1 (1-3) & 41 & 6 & $14.60 \%$ \\
\hline $\mathrm{N} 2(>3)$ & 37 & 11 & $29.30 \%$ \\
\hline
\end{tabular}


Citation: Jauhri M, Gupta V, Shokeen Y, Minhas S, Bhalla S, et al. (2017) KDR Mutation: A High-Frequency Rare Mutation and its Correlation with other Somatic Mutations in Indian Colorectal Cancer Patients. Next Generat Sequenc \& Applic 4: 148. doi: $10.4172 / 2469-9853.1000148$

Page 3 of 7

\begin{tabular}{|l|l|l|l|}
\hline \multicolumn{5}{|c|}{ Size of Tumor } \\
\hline$<5 \mathrm{~cm}$ & 35 & 13 & $37.10 \%$ \\
\hline$\geq 5 \mathrm{~cm}$ & 77 & 9 & $11.70 \%$ \\
\hline
\end{tabular}

Table 1: Clinical characteristics of 112 colorectal cancer patients.

(28.3\%) while the left side only contained $17.4 \%$ mutations. Our study reports higher number of KDR mutations in Stage III (31\%) as compared to other stages I and II (19.23\%) and IV (7.31\%). The T2 stage of depth of invasion with KDR mutation accounts for $37.5 \%$ of total patients with T2 stage. KDR mutations were found to be in greater number in patients with lymph node metastasis $(27.3 \%)$ as compared to liver metastasis (8\%).

The right side of colon might be linked to KDR mutations due to occurrence of higher frequency of KDR mutations in Right side

\section{Type of Mutation}

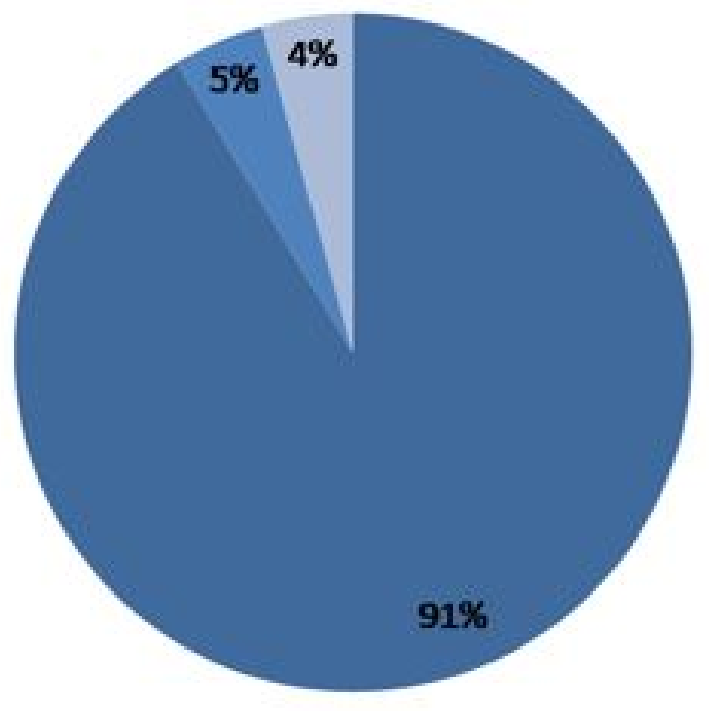

p.Q472H

p.A248G

p.G873R

Figure 1: Distribution of type of KDR mutations.

However, our study does not report any statistically significant association of KDR mutation with clinical parameters. In our knowledge, this is the first study targeting at KDR gene mutation in colon cancer using NGS. Out of the mutations in these 48 genes, mutation in KDR was found to be present in $19.6 \%$ patients. This is a comparatively high frequency with respect to rarely occurring mutations in other genes. KDR gene was found to contain three types of substitution mutations p.Q472H, p.A248G and p.G873R. The distribution of these mutations among patients is given in Figure 1. 21 patients out of 22 had p.Q472H out of which one had two mutations including p.Q472H and p.A248G. 1 out of 22 patients was reported to have p.G873R type of mutation.

Upon correlation analysis of the 48 genes, we found significant correlation of KDR with six other genes: KRAS $(p=0.026)$, APC $(\mathrm{p}=0.033), \operatorname{PTEN}(\mathrm{p}=0.003), \operatorname{ERBB} 4(\mathrm{p}=0.008)$, EGFR $(\mathrm{p}=0.036)$, and NOTCH1 $(\mathrm{p}=0.029)$ where KRAS, APC, PTEN, ERBB4, EGFR and NOTCH1 occurred in common with 12, 11, 10, 4, 3 and 2 out of 22 patients having KDR mutations respectively. Figure 2 shows the distribution of mutations among patients. 
Citation: Jauhri M, Gupta V, Shokeen Y, Minhas S, Bhalla S, et al. (2017) KDR Mutation: A High-Frequency Rare Mutation and its Correlation with other Somatic Mutations in Indian Colorectal Cancer Patients. Next Generat Sequenc \& Applic 4: 148. doi:

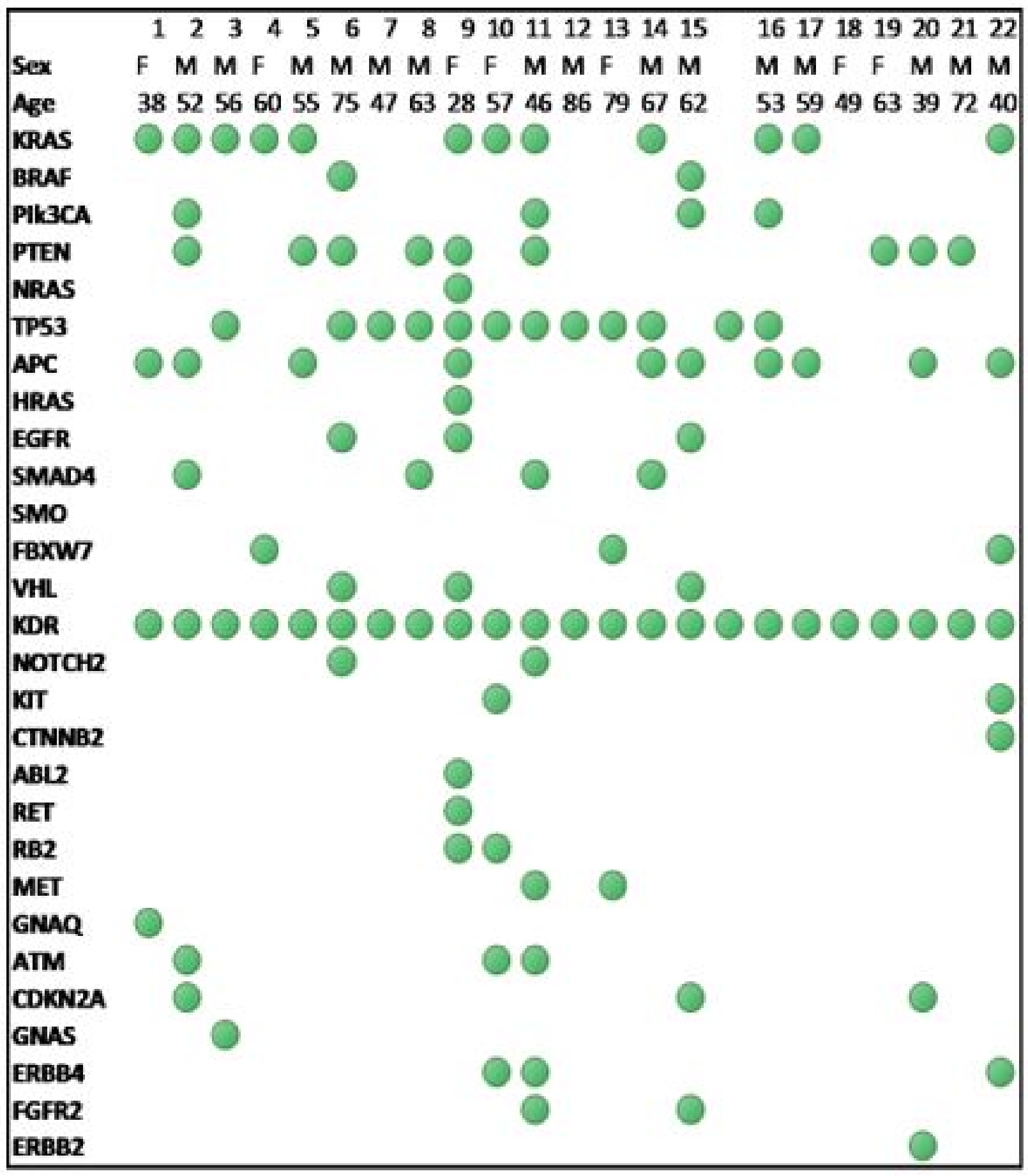

Figure 2: Co-existence of other gene mutations in patients with KDR mutation.

Also, 12 cases of TP53 mutations occurred in coexistence with KDR mutations but the correlation was not of clinical significance. Other genes of the panel did not occur in coexistence with KDR in even a single patient. Figure 3 shows significantly associated double mutations with KDR. 
Citation: Jauhri M, Gupta V, Shokeen Y, Minhas S, Bhalla S, et al. (2017) KDR Mutation: A High-Frequency Rare Mutation and its Correlation with other Somatic Mutations in Indian Colorectal Cancer Patients. Next Generat Sequenc \& Applic 4: 148. doi: $10.4172 / 2469-9853.1000148$

Page 5 of 7

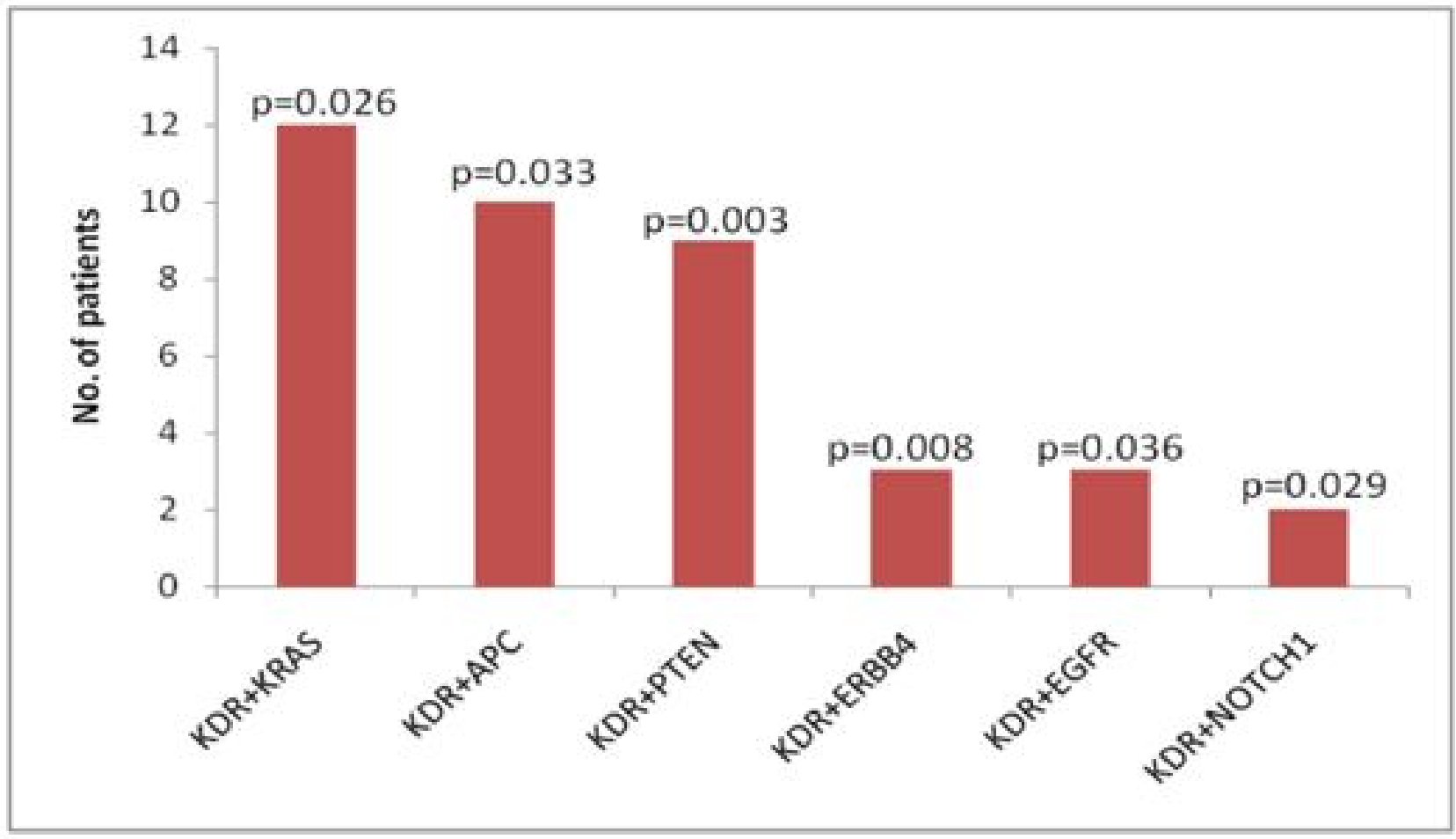

Figure 3: Significantly associated double mutations with KDR. X-axis represents number of patients with double mutations; Y-axis represents double mutations.

\section{Discussion}

Colorectal cancer is widely known to be associated with genetic mutations. These genetic mutations usually contribute to one or the other biological pathway involved in carcinogenesis. Pathways involved in development of CRC majorly include Wnt, MAPK, PI3K, mTOR, VEGF, cAMP, p53 and others (KEGG Pathway Database). But out of all these pathways, VEGF pathway plays a major role in development and progression of CRC by the process of angiogenesis. Vascular Endothelial Growth Factor Receptor (VEGFR), also known as KDR (Kinase Domain Receptor) is a protein involved in functioning of VEGF pathway. Mitogens like VEGF-A bind to KDR and signal cell proliferation and angiogenesis.

Thus, mutations in KDR have been previously found to play a role in increasing cell proliferation and angiogenesis. This brings forth the significance of studies conducted to find association between mutations in KDR and clinicopathological parameters as clinically significant results would help open avenues for new drug formulations. In the light of this, activating mutations in KDR gene have been found to be associated with increased KDR protein expression in human angiosarcoma but it has not been found to be correlated with histological type or grade. Despite no significant correlation with the histology, the same study has been successful in bringing forward the frequency of KDR mutation among the patients under study. A $10 \%$ mutation frequency of KDR gene has been reported [8]. Another study reported a frequency of $38 / 123$ patients (30.9\%) with mutation in KDR gene in Malignant Pleural Mesothelioma [9]. It is noteworthy to mention here that, a study on non-small cell lung cancer reported high $\mathrm{Q} 472 \mathrm{H}$ type of KDR mutation number in $33.3 \%$ patients [10] and in another study, it has been suggested to play a role of proto-oncogene in Lung Adeno-carcinoma [11]. Therefore, a high frequency (19.6\%) of KDR mutation among the patients in our study may also indicate its role in developing cancer. Interestingly, our study also reports high occurrence of p.Q472H (21 out of 22 patients) type of mutation which highlights the role of $\mathrm{p} . \mathrm{Q} 472 \mathrm{H}$ mutation in the KDR gene. This is also substantiated by the study in which p.Q472H has been found to be mutated in large intestine carcinomas [12].

Mutation in KDR gene was found to be higher in Lymph node metastasis as compared to liver metastasis in our study. This supports the study in which expression of VEGF-C, (which upon proteolytic processing binds to VEGFR-2 (KDR)), was significantly associated with lymph node metastasis in gastric carcinoma [13]. But on the other hand, this result is also in contrast to a previous study in which $27 \%$ cases with KDR mutations were associated with Colorectal Liver metastasis [12]. This difference might be present due to the small patient population size in our study. KDR gene has been associated with VEGF signaling pathway which is also linked with PIK3CA and PTEN proteins. Our study reports a significant correlation between KDR and PTEN gene mutations which suggests their associated role in development of colon cancer. However, this is in contrast to one study conducted in Small cell lung cancer patients in which PI3K/AKT/ mTOR pathway status did not correlate with KDR gene [14]. This difference might be present due to different cancer groups and the small number of patients studied. Furthermore, in a recently reported case study [15], KDR mutation, KRAS mutation and APC mutation were found together in a patient of colon cancer although no clinical significance was reported for their correlation. This case study collectively with our results indicates that KRAS and KDR mutation 
Citation: Jauhri M, Gupta V, Shokeen Y, Minhas S, Bhalla S, et al. (2017) KDR Mutation: A High-Frequency Rare Mutation and its Correlation with other Somatic Mutations in Indian Colorectal Cancer Patients. Next Generat Sequenc \& Applic 4: 148. doi: $10.4172 / 2469-9853.1000148$

Page 6 of 7

can together play a role in proliferation of tumor cells when VEGF signaling pathway proceeds via MAPK signaling route. Not only this, $\mathrm{APC}$ and KDR mutations have been independently reported to play a role in tumorigenesis of gastrointestinal cancer and colon cancer but no previous reports of correlation between APC and KDR were found [16-18]. Also, our study suggests a significant correlation between NOTCH1 and KDR (VEGFR2) but we found no literature supporting the same. However, NOTCH1 has been associated with VEGFR1 in previous studies [19]. Moreover, a discussion on NOTCH and VEGF signaling proposes a model where NOTCH1 and VEGFR2 may play symbiotic roles in angiogenesis [20]. This indicates that there might also be an association of NOTCH1 and VEGFR2 in combined development towards angiogenesis which strengthens our results. Interestingly, KDR (VEGFR2) and EGFR have been identified as potential targets for many anti-cancer therapies [21-23] but no studies could be found showing direct correlation between KDR and EGFR. The combined therapeutic targeting to treat cancer is also suggestive of their significant association in regulating cancer pathways which can be seen in our results which shows a significant correlation between mutations in EGFR and KDR genes. Not only this, drugs targeting EGFR, ERBB4 and KDR together have also been studied previously [24]. Moreover, EGFR and ERBB4 belong to the same Receptor Tyrosine Kinase family and work in close association. Thus, a correlation between EGFR and KDR mutations may also indicate the possibility of ERBB4 and KDR mutation. But the results need to be validated by performing assays in larger number of patients.

However, high KDR protein expression has been previously reported in many cancer studies projecting its role in tumorigenesis and angiogenesis $[8,25,26]$. Overall, this can mean that the KDR mutation leads to high expression of KDR protein. In addition, KDR gene mutation has been previously reported to play a therapeutic role using Regorafenib in metastatic colon cancer [14] as well as in Nonsmall cell lung cancer [27]. On the contrary, it has also been reported to occur as an adverse effect of Ramucirumab therapy [28] and may lead to side effects when used with FGFR inhibitors for treatment. Additionally, COSMIC database suggests its association with Sorafenib. So, mutation in KDR gene is therapeutically important and any association with clinical parameters or pathways can further help in embarking research focused on drugs targeting KDR. Intriguingly, KDR gene as a potential therapeutic target for angiogenesis inhibitor drugs has been reported previously [29-31].

\section{Conclusion}

Our results in combination with previous studies suggest a role for KDR in colon cancer also making it a potential therapeutic target for various drugs like Regorafenib and Sorafenib. Thus, the results from our study can be used for employing targeted therapy in patients with KDR mutations. Besides, it might also be associated with one or more clinical parameters which can help prognostic studies. Significant correlations of KDR mutation with other gene mutations - KRAS, APC and PTEN has shown a new possibility for association of signaling pathways in development of tumors and angiogenesis in Colorectal Cancer. Further studies on KDR and other genes' expression and polymorphisms in colorectal cancer using a larger patient cohort are required for the validation of these results.

\section{Acknowledgement}

The study was funded by Merck Serono India. The authors express their gratitude to Strand Life Sciences for their support during data preparation and to Mrs. Parul Takkar for her help in statistical analysis.

\section{Permissions}

\section{Ethics approval}

Institutional and/or National Committee ethical standards were followed throughout the study. All procedures involving human participants in the study were in accordance with the 1964 Helsinki declaration and its later amendments or comparable ethical standards. This article does not contain any studies with animals performed by any of the authors.

\section{Informed consent}

Informed consent was obtained from all individual participants included in this study.

\section{References}

1. International Agency for Research of Cancer, WHO (2012) Fact sheets of cancer.

2. KEGG Pathway Database (2005) Kanehisa Laboratories, Japan.

3. Takahashi Y, Kitadai Y, Bucana CD, Cleary KR, Ellis ML (1995) Expression of vascular endothelial growth factor and its receptor, KDR, correlates with vascularity, metastasis, and proliferation of human colon cancer. Cancer Res 55: 3964-3968.

4. Zhang X, Ge YL, Zhang SP, Yan P, Tian RH (2014) Down regulation of KDR expression induces apoptosis in breast cancer cells. Cell Mol Biol Lett 19: 527-541.

5. Arrington A, Heinrich E, Lee W, Duldulao M, Patel S, et al. (2012) Prognostic and predictive roles of KRAS mutation in colorectal cancer. Int J Mol Sci 13: 12153-12168.

6. Barras D (2015) BRAF mutation in colorectal cancer: An update. Biomark Cancer 7: 9-12.

7. Cathomas G (2014) PIK3CA in colorectal cancer. Front Oncol 4: 1-4.

8. Antonescu CR, Yoshida A, Guo T, Chang NE, Zhang L, et al. (2009) KDR activating mutations in human are sensitive to specific kinase inhibitors. Cancer Res 69: 7175-7179.

9. Lo Iacono M, Monica V, Righi L, Grosso F, Libener R, et al. (2015) Targeted next-generation sequencing of cancer genes in advanced stage malignant pleural mesothelioma: A retrospective study. J Thorac Oncol 10: 492-499.

10. Masago K, Fujita S, Muraki M, Hata A, Okuda C, et al. (2015) Nextgeneration sequencing of tyrosine kinase inhibitor-resistant non-smallcell lung cancers in patients harboring epidermal growth factor-activating mutations. BMC Cancer 15: 908.

11. Tanoue LT (2010) Somatic mutations affect key pathways in lung adenocarcinoma. Yearbook of Pulmonary Disease 115-117.

12. Lee SY, Haq F, Kim D, Jun C, Jo HJ, et al. (2014) Comparative genomic analysis of primary and synchronous metastatic colorectal cancers. PLoS one 9: e90459.

13. Onogawa S, Kitadai Y, Amioka T, Kodama M, Cho S, et al. (2005) Expression of vascular endothelial growth factor (VEGF)-C and VEGF-D in early gastric carcinoma: correlation with clinicopathological parameters. Cancer Lett 226: 85-90.

14. Cheng H, Shcherba M, Pendurti G, Liang Y, Piperdi B, et al. (2014). Targeting the PI3K/AKT/mTOR pathway: Potential for lung cancer treatment. Lung Cancer Manag 3: 67-75. 
Citation: Jauhri M, Gupta V, Shokeen Y, Minhas S, Bhalla S, et al. (2017) KDR Mutation: A High-Frequency Rare Mutation and its Correlation with other Somatic Mutations in Indian Colorectal Cancer Patients. Next Generat Sequenc \& Applic 4: 148. doi: $10.4172 / 2469-9853.1000148$

Page 7 of 7

15. Loaiza-Bonilla A, Jensen CE, Shroff S, Furth E, Bonilla-Reyes PA, et al. (2016) KDR mutation as a novel predictive biomarker of exceptiona response to regorafenib in metastatic colorectal cancer. Cureus 8: e478.

16. Morin PJ, Vogelstein B, Kinzler KW (1996) Apoptosis and APC in colorectal tumorigenesis. Proc Natl Acad Sci USA 93: 7950-7954.

17. Taketo MM (2006) Wnt signaling and gastrointestinal tumorigenesis in mouse models. Oncogene 25: 7522-7530.

18. Warren RS, Yuan H, Matli MR, Gillett NA, Ferrara N (1995) Regulation by vascular endothelial growth factor of human colon cancer tumorigenesis in a mouse model of experimental liver metastasis. J Clin Invest 95: 1789-1797.

19. Paiva T, De Jesus V, Marques R, Anastácio Da-Costa AA, Petaccia DeMacedo M, et al. (2015) Angiogenesis-related protein expression in bevacizumab-treated metastatic colorectal cancer: NOTCH1 detrimental to overall survival. BMC Cancer 15: 643 .

20. Hellstrom M, Phng L, Gerhardt H (2007) VEGF and Notch Signaling. Cell Adh Migr 1: 133-136.

21. Ciardiello F, Troiani T, Bianco R, Orditura M, Morgillo F, et al. (2006) Interaction between the epidermal growth factor receptor (EGFR) and the vascular endothelial growth factor (VEGF) pathways: A rational approach for multi-target anticancer therapy. Ann Oncol 7: vii109-114.

22. Traxler P, Allegrini PR, Brandt R, Brueggen J, Cozens R, et al. (2004) AEE788: A dual family epidermal growth factor receptor/ErbB2 and vascular endothelial growth factor receptor tyrosine kinase inhibitor with antitumor and antiangiogenic activity. Cancer Res 64: 4931-4941.

23. Yokoi K, Thaker PH, Yazici S, Rebhun RR, Nam DH, et al. (2005) Dua inhibition of epidermal growth factor receptor and vascular endothelial growth factor receptor phosphorylation by AEE788 reduces growth and metastasis of human colon carcinoma in an orthotopic nude mouse model. Cancer Res 65: 3716-3725.
24. Pan Y, Xu Y, Feng S, Luo S, Zheng R, et al. (2012) SKLB1206, a novel orally available multikinase inhibitor targeting EGFR activating and T790M mutants, ErbB2, ErbB4, and VEGFR2, displays potent antitumor activity both in vitro and in vivo. Mol Cancer Ther 11: 952-962.

25. Teicher BA (2012) Searching for molecular targets in sarcoma. Biochem Pharmacol 84: 1-10.

26. Phillips HS, Kharbanda S, Chen R, Forrest WF, Soriano RH, et al. (2006) Molecular subclasses of high-grade glioma predict prognosis, delineate a pattern of disease progression and resemble stages in neurogenesis. Cancer Cell 9: 157-173.

27. Veldore VH, Patil S, Satheesh CT, Shashidhara HP, Tejaswi R, et al. (2015) Genomic profiling in a homogeneous molecular subtype of non-small cell lung cancer: An effort to explore new drug targets. Indian J Cancer 52: 243-248.

28. Lim YH, Odell ID, Ko CJ, Choate KA (2015) Somatic p.T771R KDR (VEGFR2) Mutation arising in a sporadic angioma during ramucirumab therapy. JAMA Dermatol 151: 1240-1243.

29. Gavilondo JV, Hernández-Bernal F, Ayala-Ávila M, De La Torre AV, De La Torre J, et al. (2014) Specific active immunotherapy with a VEGF vaccine in patients with advanced solid tumors. Results of the CENTAURO antigen dose escalation phase I clinical trial. Vaccine 32: 2241-2250.

30. Li Q, Wang Y, Zhang L, Chen L, Du Y, et al. (2016) Naringenin exerts anti-angiogenic effects in human endothelial cells: Involvement of ERRa/ VEGF/KDR signaling pathway. Fitoterapia 111: 78-86.

31. Yu DH, Lu Q, Xie J, Fang C, Chen HZ (2010) Peptide-conjugated biodegradable nanoparticles as a carrier to target paclitaxel to tumor neovasculature. Biomaterials 31: 2278-2292. 